\title{
Mar del Plata: de la villa balnearia al balneario de masas. Una metáfora de la sociedad argentina ${ }^{1}$
}

Juan Carlos Torre

A la memoria de Toto

\section{Introducción}

Los cambios sociales de la Argentina moderna fueron dejando su marca en Mar del Plata como sobre una tela en blanco. Desde la villa balnearia construida a fines del siglo XIX para servir de solar veraniego de las clases altas hasta la capital del turismo de masas en la que se convierte en las décadas de 1950 y 1960, con la proliferación de rascacielos y hoteles sindicales, la evolución del balneario describe un itinerario que acompaña, a cada momento, las transformaciones sociales de la Argentina. Su historia se confunde con la de una sociedad que, con el paso del tiempo, va ampliando las oportunidades de bienestar social y, con ellas, hace accesible a cada vez más argentinos la experiencia de unas vacaciones junto al mar. Vistas retrospectivamente, las mutaciones de Mar del Plata condensan, en forma ejemplar, el impulso igualitario que movilizó a lo largo de los años a sucesivas generaciones y que dio sostén a un proyecto social fuera de lo común en el mundo: un balneario de todos, donde los más diversos sectores sociales pudiesen compartir el verano en las mismas playas.

\section{La villa balnearia}

Consultando los diarios de enero de 1888 nos enteramos que estaba por abrir sus puertas el primer gran hotel de Mar del Plata, el Bristol Hotel

\footnotetext{
${ }^{1}$ Este texto es la hoja de ruta del libro que escribí con Elisa Pastoriza. Con el título Mar del Plata, un sueño de los argentinos será publicado por la Editorial Edhasa a fines de 2019.
} 
«[En] Buenos Aires no se habla de otra cosa.En los grandes salones, en el teatro, en los paseos, en todas partes, el tema era el mismo. Decíase y con razón que el nuevo hotel estaba destinado a ser el preferido de la elite argentina (...) Cien cartas de invitación enviadas por el fundador del Bristol, el señor JoseLuro, circularon entre personas de alta figuración social. $\mathrm{Y}$ el viernes 7 de enero a la noche la flamante locomotora del Ferrocarril del Sur ponía en movimiento sus potentes músculos de acero y haciendo rechinar sus ejes y palancas se lanzaba hacia el sur, llevando su carga humana, ansiosa de agradables sorpresas» ${ }^{2}$

La primera sorpresa de los viajeros no fue nada agradable y en verdad, tampoco fue una sorpresa: el tren arribó a destino en un día lluvia y viento tan característico del clima destemplado del verano en Mar del Plata. Por la crónica publicada en La Nación nos enteramos de que

«El desembarco en la estación de Mar del Plata fue un completo desastre. Figuraos a nuestras bellas acompañantes con sus frescos trajes veraniegos, sombreros de tules y botines de género, corriendo desde los vagones a los coches que aguardaban en la estación sobre un piso fangoso. La lucha por los carruajes tenía forzosamente que ser, como fue, sin condiciones Todas las reglas de la galantería quedaron por tierra: había necesidad de salvarse del chubasco. Por fin llegamos al hotel, siempre bajo la lluvia, y tomamos las habitaciones por asalto» ${ }^{3}$

El elenco de los viajeros estaba encabezado por Carlos Pellegrini, vicepresidente de la nación, e incluía al gobernador de la provincia de Buenos Aires, los directores de los principales diarios porteños y un selecto grupo de amistades y parientes del dueño del hotel. Para todos ellos y una vez que salieron de las habitaciones con sus mejores galas, el gran comedor del Bristol abrió sus puertas por primera vez. El banquete de recepción, al cuidado de los 24 cheffs, cocineros y mozos contratados en Paris, seguramente contribuyó a que olvidaran las tribulaciones de la llegada mientras saboreaban los platos de la cocina francesa.

Días después, en la fiesta que se realizó en los salones del hotel, Pellegrini, quién se convirtió bien pronto en animador principal de la

${ }^{2}$ El Diario, 15/02/1908.

${ }^{3}$ La Nación, 09/01/1888. 
vida de balneario, hizo un brindis profetizando un grandioso futuro para Mar del Plata. Su profecía se cumplió con creces porque, según anotó un cronista unos años más tarde, «el veraneo en Mar del Plata se ha convertido en un rito obligado y complemento indispensable de la vida aristocrática de Buenos Aires». ${ }^{4}$ Agreguemos que las comodidades que ofreció el Bristol Hotel también recibieron su premio. En febrero de 1907 el corresponsal de un diario dictaminó que «la vida de los veraneantes está concentrada en el Bristol Hotel». ${ }^{5}$

Construido con materiales espléndidos y con detalles de confort en gran parte importados de Europa, el Bristol Hotel hizo posible que la elegancia y el lujo se volvieran compatibles con pasar los meses de verano en las playas. Hizo posible, en fin, que la elite porteña pudiera seguir haciendo ostentación de su riqueza al abandonar temporalmente Buenos Aires. En los hechos, el hotel se convirtió en su hogar sustituto durante el verano; por sus instalaciones familias enteras se movían a sus anchas en la cita anual a orillas del Atlántico, en compañía de los mismos rostros y apellidos familiares.

Confortable y selecto, el Bristol Hotel ofreció a la alta sociedad un servicio de primer orden, me refiero a la iniciación a los usos y costumbres del ocio distinguido. Al igual que el Jockey Club en Buenos Aires, el hotel tuvo por misión promover, según palabras de Alberto del Solar, «el refinamiento de nuestras clases elevadas mediante el fomento de los hábitos de sociedad». ${ }^{6}$ Para Alberto del Solar, un chileno hombre de mundo, y también para sus contemporáneos, los hábitos de sociedad eran los de las grandes referencias socioculturales de la época, las aristocracias de Inglaterra y Francia. Fue así que, en los salones del Bristol, la sociabilidad veraniega se desenvolvió regida por los ritmos de una pedagogía elaborada y exigente.

Embarcadas en un acelerado curso de auto-educación, las familias de la alta sociedad se inclinaron sin reservas ante la dictadura de los convencionalismos de moda que pautaban la hora del baño, las comidas, los entretenimientos e imponían un atuendo elegante estándar para cada actividad. En 1922, Francisco Granmontagne, un periodista español con gran recorrido en la Argentina del 1900, al echar una mirada retrospectiva sobre Mar del Plata sostuvo:

${ }^{4}$ BonsiuKurile, (seudónimo de Benjamin Solari) «La vida en Mar del Plata, pinceladas realistas», 1907, p.61.

${ }^{5}$ El Censor, 08/02/1889.

${ }^{6}$ El Diario, 15/02/1908. 
«No existe en el país un centro que haya tenido mayor influencia que el Bristol en la transformación de los hábitos argentinos. En su comedor, semejante a la nave de una catedral, se iniciaron las formas de la convivencia elegante. Fue apenas inaugurado como una cátedra de refinamiento de las costumbres. Los toscos salían de allí, al mes, transformados, como trogloditas pasados por Versalles» ${ }^{7}$

La iniciación a los usos y costumbres del ocio distinguido vino acompañada con el despliegue de un tren de vida suntuoso y, junto con él, por la competencia en brillar a la hora de ostentarlo. Observando ese espectáculo, un visitante extranjero señaló:

«En el Bristol la única ocupación desde la mañana hasta la hora de acostarse consiste en hacer pompa del mayor número de toilettes, si de las señoras se trata, y también si de hombres, los que pasarán con fama de elegantes según la media docena de corbatas, pantalones y jacquet que en un solo día hayan hecho gala en los salones del hotel» ${ }^{8}$

Al cabo de unos años, tener una residencia en Mar del Plata se convirtió en una obligación mundana para los ricos de Buenos Aires. Y no ahorraron pesos ni se privaron de excesos en su afán por competir en el derroche del lujo y la originalidad de sus mansiones de verano. En forma simultánea procuraron acondicionar las orillas del mar en sintonía con su estilo de vida ampuloso. Con el aporte de fondos públicos y privados financiaron la construcción de la Rambla Bristol, una obra que, en la época, sobresalió por sus grandes dimensiones y su esplendor arquitectónico entre los balnearios del mundo. Inaugurada en 1913, pronto fue conocida como la rambla francesa, era un edificio de 400 metros de largo y 45 metros de ancho, con cabinas de baños y negocios, en el que sobresalía el paseo al aire libre, con vista al mar, que ofrecía la plataforma para un verdadero desfile ceremonial. A las doce de la mañana y a las seis de la tarde la rambla era un ir y venir de gente orquestado por un estricto protocolo. Las personas iban erguidas, casi sin mirarse unas a otras; era de mal gusto interrumpir la marcha para conversar, los intercambios debían ser breves y poco efusivos. Estos gestos y actitudes estaban dictados por lo que los círculos encumbrados entendían que debían ser los comportamientos distinguidos en lugares públicos. Estas cami-

${ }^{7}$ La Prensa, 19/02/1922.

${ }^{8}$ Jules Huret, «En Argentine», Vol.II, 1913. 
natas por la Rambla Bristol tenían un doble propósito: facilitar a los miembros de la alta sociedad verse a sí mismos como formando parte de un círculo refinado y a la vez exhibirse como tales ante el conjunto de la sociedad. Esa puesta en escena tenía una tendencia hacia la sobreactuación con un propósito y éste era: diferenciarse del talante más jocundo y menos acartonado, tan característico de los nuevos veraneantes que comenzaron a llegar a Mar del Plata.

A 400 kilómetros de Buenos Aires, Mar del Plata irradiaba una atracción irresistible como símbolo que era de la consagración social en la Argentina de entonces. Era esperable que los miembros más afortunados de las clases medias dirigieran su atención a ella. A partir dela primera década del siglo la demanda de vacaciones junto al mar se filtró desde la cima hasta la zona media de la pirámide social alimentada por una aspiración: la persona o la familia que veraneaba en Mar del Plata adquiría con ello un aire de distinción entre sus parientes y amistades. Como consecuencia, no hubo esfuerzo o sacrificio al que se negaran para viajar a la costa y conquistar ese trofeo social.

Una nota en la revista PBT de 1907, bajo el título «A la costa a toda costa» describía las vicisitudes de un funcionario público en su esfuerzo por llevar a su familia a Mar del Plata: contrajo hipotecas, soportó peleas con su esposa. La razón: que ella pudiera lucir sus nuevos vestidos en el balneario. Estos tormentosos preparativos en las vísperas del verano - destacaba la revista- eran comunes en la periferia de la alta sociedad donde «el qué dirán tiene más fuerza que una carga de caballería». ${ }^{9}$ Diez años después leemos en Caras y Caretas una historia parecida. «Esta noche nos ausentamos a Mar del Plata. Al final realizo la aspiración de toda mi vida», ${ }^{10}$ así se confesaba el personaje de una nota apenas ficticia donde se hacía el inventario de las deudas con el carnicero, el cobrador de luz y otros más en las que incurría un pequeño comerciante para que su esposa y su hija pudiesen veranear bajo el mismo cielo que las familias de la alta sociedad.

Para atender al flujo de estos nuevos veraneantes proliferaron los alojamientos más modestos, y ello permitió que un cronista extranjero pudiese escribir:

«Mar del Plata tiene algo digno de encomio: que es accesible a todos los bolsillos, tanto del millonario que va a instalarse en medio e un

${ }^{9}$ PBT, $\mathrm{N}^{\circ} 73$, febrero de 1907.

${ }^{10}$ Caras y Caretas, 21/01/1928. 
lujo asiático en el Bristol Hotel, cuando al modesto empleado de comercio, que lleva su equipaje a un hotel de cuarto orden, para después aparecer en la Rambla con aire de rentista en busca de distracción» ${ }^{11}$

Esta metamorfosis del paisaje social trastocó la escenografía del veraneo marplatense. Durante los primeros años su centro de gravedad estuvo ocupado por la figura dominante de los sectores encumbrados; por detrás de ella, en el fondo, se podía vislumbrar un mundo más popular. Ahora ese mundo más popular estaba dando un paso al frente y ganaba mayor visibilidad. El corresponsal de un periódico porteño pudo escribir en 1917:

«En otros años, cuando Mar del Plata era el centro de unas pocas familias adineradas la presencia de una persona que no formara parte de ese núcleo llamaba la atención. Ahora la situación ha cambiado, y llega aquí todo el que tiene deseo de hacerlo y se encuentra a gusto, sin llamar la atención, llevando una vida tranquila y agradable» ${ }^{12}$

Esta creciente visibilidad de los nuevos veraneantes introdujo un motivo de preocupación: que Mar del Plata se democratizara. Las voces de alarma se hicieron oír bien pronto. El corresponsal del diario La Razón escribió en 1911: «Hay una perspectiva que no escapa a la observación del cronista y es que, siguiendo su actual desarrollo, Mar del Plata se democratice. Y, en consecuencia, que la alta sociedad, que le gusta encerrase en círculos tan estrechos como concéntricos, se aleje o se refugie en sus chalets». ${ }^{13}$ Hacia 1916 ya no hay dudas: para el corresponsal de Caras y Caretas el vaticinio hecho cinco años antes por su colega de La Razón se ha cumplido: «Si bien es cierto que a la rambla la frecuentan algunas eminencias sociales, la mayoría de éstas la abandona a la inmensa marea de veraneantes que afluye desde todos los ámbitos. Las elegantes porteñas se refugian en sus lujosas residencias porque temen el arenal movedizo y traidor que forma la superficie social de Mar del Plata». ${ }^{14}$

${ }^{11}$ F.Scardin, citado en Francis Korn, «Buenos Aires. Mundos Particulares», 2004, p.31.

${ }^{12}$ La Razón, 23/01/1917.

${ }^{13}$ La Razón, 19/01/1911.

${ }^{14}$ Caras y Caretas, 04/01/1916. 
El corresponsal de Caras y Caretas no podía haber sido más certero al llamar la atención al «arenal movedizo y traidor» que erosionaba los cimientos de la villa balnearia y ponía bajo asedio los lugares y las prácticas de la alta sociedad. El repliegue de la alta sociedad a sus residencias familiares, esto es, a ámbitos cuyas puertas estuvieran rigurosamente vigiladas, tuvo una primera víctima: la declinación del que fuera el centro de la vida social del balneario, el Bristol Hotel.

Los cambios que estaba experimentando Mar del Plata proyectaron sus efectos más allá de la esfera de la sociabilidad. Gracias a las garantías al ejercicio del sufragio introducidas por la Ley Sáenz Peña de 1912 se produjo un sorpresivo viraje en el control político de la villa balnearia: los comicios de 1920 pusieron un socialista, Teodoro Bronzini, en la conducción del municipio. Explicando a sus conocidos este inesperado desenlace una dama de la alta sociedad escribió:

«Para el visitante que no se encuentra interiorizado de la vida política de Mar del Plata, que ignora cómo está formada su población, resulta algo incomprensible que una ciudad, por excelencia aristocrática, llena de suntuosos y elegantes palacios, esté gobernada por socialistas. Es que los habitantes estables son la inmensa cantidad de obreros del puerto, pintores, albañiles, cuidadores de fincas, etcétera, que trabajan todo el año y residen en la ciudad. La gente acaudalada que tiene su chalet no vive en Mar del Plata y por lo tanto no vota» ${ }^{15}$

Esta explicación merece una corrección: el partido socialista recibió también votos de las ascendientes clases medias de origen inmigratorio que poblaban la ciudad y tenían un rol sobresaliente en la industria del veraneo.

Desmintiendo los temores de los antiguos veraneantes ante el surgimiento de la comuna roja, los socialistas no descuidaron la industria del veraneo. Pero le agregaron un nuevo componente. En 1925 formaron una comisión de propaganda y le asignaron la tarea de desvirtuar, cito sus propias palabras, «el viejo prejuicio que alienta todavía mucha gente según el cual no puede llegar a estas playas sino la parte más rica y afortunada del país». ${ }^{16}$ Para ello lanzaron una campaña en favor de trenes de segunda clase, exhortaron a los hoteles y pensiones a que reduje-

${ }^{15}$ Virginia Paul de Guevara «Las Chicas de Mar del Plata», 1924, p.50.

${ }^{16}$ Boletín Municipal de Mar del Plata, julio de 1925, p.l. 
ran los precios e imprimieron miles de folletos distribuidos en todo el país exaltando las ventajas del balneario. Si hasta allí la afluencia de veraneantes había sido el resultado de múltiples decisiones individuales ahora era el objetivo de una política pública, que comportó toda una innovación en la época: hacer que la villa balnearia fuese accesible a las aspiraciones de las personas de condición más modesta.

Los socialistas estuvieron en la intendencia de Mar del Plata unos diez años, hasta que en 1929 con un pretexto ocasional el municipio fue intervenido. La terminación abrupta de esa experiencia no interrumpió el proceso de apertura del antiguo balneario aristocrático. En 1928 las fuerzas vivas de la ciudad -dueños de comercio y hoteles, empresarios de la construcción- y la participación de los propios socialistas, fundaron una Comisión de Fomento que tendría una gran gravitación en los años por venir. La consigna de la comisión fue cabal expresión de los nuevos tiempos: «Por la Democratización del Balneario».

Que los tiempos eran nuevos lo certificó un artículo en la revista El Hogar de ese mismo año 1928. En él leemos:

«Hace algunos años, si a cualquiera se le hubiese ocurrido hablar del gran balneario argentino como lugar de fraternización democrática, donde se alternan y se confunden las clases sin molestarse, se le habría calificado de tonto...Entonces era idea admitida que Mar del Plata era como una perla ofrecida por el Atlántico a los aristócratas y magnates. Hoy semejante afirmación sería sencillamente absurda. Mar del Plata es el balneario de todos, del potentado y del burgués, del empleado y del obrero» ${ }^{17}$

Podría sin duda discutirse la justeza de este diagnóstico pero sería un ejercicio fútil porque sus efectos eran reales desde la perspectiva del alto mundo social. En 1928 un antiguo veraneante entrevistado en la misma revista se lamentó: «En mis tiempos bastaba asomarse al gran comedor del Bristol para tener una sensación que halagaba nuestra vanidad social. Todos nos conocíamos, cada mesa era como un palco de la vieja Opera. Mar del Plata era nuestra. Hoy, en cambio, cuando arriesgo a pasear por la rambla se me ocurre que estoy en otra parte». ${ }^{18}$

Reiteremos: que los tiempos eran nuevos lo sabemos al constatar que hacia el final de la década de 1920 varias familias de la alta sociedad

${ }^{17}$ El Hogar, 16/03/1928.

${ }^{18}$ El Hogar, N ${ }^{\circ} 100,1928$. 
abandonaron en favor de los nuevos veraneantes la Playa Bristol, el sitio tradicional de la vida elegante del balneario e iniciaron el éxodo hacia el sur, instalándose más allá del Cabo Corrientes. Quién encabezó esa forzada mudanza «para evitar», según nos dice un cronista, «la pleamar de las multitudes que se vuelcan en los tramos centrales del balneario», ${ }^{19}$ fue el presidente Marcelo Torcuato de Alvear, que hizo construir en Playa Grande una residencia para su esposa y la transformó en la avanzada de la nueva zona selecta de Mar del Plata.

En 1932 la comisión a la que ya aludimos, formada por las fuerzas vivas del balneario, colocó este cambiante panorama en perspectiva y señaló:

«Veinte o treinta familias componían la totalidad del mundo veraniego. Un pequeño industrial, un modesto comerciante, un empleado, que en los viejos tiempos le hubiesen hablado de Mar del Plata, estiraría los labios en un gesto típico de admiración, de sueño irrealizable y diría iMar del Playa: eso está solamente al alcance de los privilegiados! Hoy por fortuna, los tiempos han cambiado. Mar del Plata se ha puesto a tono con las prácticas democráticas que deben ser la norma de nuestras costumbres. Si todavía subsiste un grupo selecto que se va batiendo en retirada ante el avance igualitario y añora los tiempos de antaño, hoy en tenemos en cambio una Mar del Plata pletórica de vida. Hay que llevar al conocimiento de toda la república que Mar del Plata es finalmente accesible a las gentes modestas $»^{20}$

\section{Hacia el balneario de masas}

La convocatoria a los argentinos lanzada desde Mar del Plata tuvo un efecto previsible: la fuerza de las aspiraciones no es independiente de que se las piense legítimas y se las considere viables. En el lapso de diez años los turistas se multiplicaron por seis. Fue contra este telón de fondo que a finales de la década de 1930 el balneario fue hecho prácticamente de nuevo. Quién dirigió esa transformación fue el gobernador conservador Manuel Fresco, admirador de Mussolini y cultor del fraude electoral. Él fue el promotor de un vasto programa de obras públicas que tendría

${ }^{19}$ La Nación, 03/02/1927.

${ }^{20}$ «Los baños del mar y la democratización de las costumbres», Mar del Plata Anuario, 1931-1932. 
en el engrandecimiento de Mar del Plata su realización más ostensible. Tres fueron sus principales iniciativas. La primera, la pavimentación del tramo de la Ruta 2 entre Dolores y Mar del Plata en 1938; ello permitió que el viaje de los turistas, hasta entonces en tren, se hiciera también en automóviles y ómnibus. La segunda fue la construcción de modernas instalaciones en Playa Grande para ofrecer un lugar de recepción acorde al traslado de la elite veraneante. La tercera fue la más monumental de todas porque tuvo la audacia de borrar el mapa a la Rambla Bristol para levantar en su lugar dos macizos edificios gemelos, el Casino y el Hotel Provincial. Quedó delineado, así, rotundo y magnífico, un nuevo dibujo urbano de Mar del Plata.

A primera vista pareció convalidar las mutaciones operadas en el paisaje social en los años previos. Sin embargo, a nuestro juicio, estuvo guiado por un proyecto más ambicioso: crear una ciudad balnearia donde todos los sectores sociales hallaran una puerta de entrada y pudiesen compartir el mismo mar y el mismo cielo pero en el que las fronteras sociales estuviesen claramente delimitadas con el objeto de facilitar la convivencia. El diario La Prensa fue bien expresivo al respecto: «El hecho de que puedan convivir cómodamente personas de las más variadas posibilidades económicas es uno de los elementos que le dan a Mar del Plata la categoría de gran ciudad balnearia». ${ }^{21}$

Esta mirada sobre Mar del Plata es del año 1944, esto es, en las vísperas de otra gran transformación del balneario, la que vendrá con los años peronistas. Vayamos al final de este período tan portador de novedades y démosle la palabra a su creador, Juan Domingo Perón. En 1954, en ocasión de la inauguración del Primer Festival de Cine de Mar del Plata, Perón afirmó:

«Hace 10 años visité Mar del Plata y en ese entonces era un lugar de privilegio, donde los pudientes del país venían a descansar los ocios de toda la vida. Han pasado 10 años. Durante ellos esta maravillosa síntesis de nuestra patria aglutina en sus playas al pueblo argentino y, en especial, a sus hombres de trabajo. Nuestro lema fue cumplir también acá. Nosotros no quisimos una Argentina disfrutada por un grupo de privilegiados sino una Argentina para el pueblo argentino» ${ }^{22}$

\footnotetext{
${ }^{21}$ La Prensa, 04/01/1944.

${ }^{22}$ Boletín Municipal de Mar del Plata, 1954, p.112.
} 
En el momento en que Perón hablaba a la multitud adicta allí reunida ya había arraigada en ella un relato de la historia del país en el cual el período anterior a su llegada al poder era asociado al predominio de los más ricos y poderosos. Por lo tanto, es probable que pocos de los que lo escuchaban hayan reparado en cuán escasamente fidedigna era la visión que Perón ofrecía de Mar del Plata a la época de su visita 10 años antes, en 1944. Para entonces, con la demolición de la Rambla Bristol en 1940 y el cierre del Bristol Hotel unos años tarde los tiempos de la villa balnearia habían sido simbólicamente clausurados y había comenzado el nuevo ciclo de la ciudad como balneario de masas. Durante la década siguiente Perón extendió, por cierto, a través del largo brazo de su política social, el alcance de las vacaciones de verano a más sectores del mundo del trabajo. Pero si quienes lo aclamaban con la vista puesta en los balcones del casino hubiesen extendido su mirada hasta los paseos y playas de Mar del Plata habrían comprobado que también él, como otros antes, exageraba a la hora de apreciar el grado de apertura social del balneario. Todavía habrían de faltar más años y más iniciativas para que los obreros llegaran a representar una proporción mayor entre los argentinos de vacaciones junto al mar. Al respecto es oportuno destacar, contra una opinión muy extendida, que los hoteles sindicales se multiplicaron en el balneario recién después de 1955, en particular, luego de la sanción de la Ley de Obras Sociales de 1970 bajo la dictadura del General Onganía. La principal iniciativa en el campo del turismo social durante los años peronistas fue la construcción de la Colonia de Vacaciones de Chapadmalal.

Ahora bien, cuando se pone en perspectiva qué fue lo que cambió en el balneario en esos años una iniciativa sobresale, el impacto de la Ley de Propiedad Horizontal aprobada en 1948, que hizo posible la propiedad de unidades individuales de departamentos. La ley y los créditos del Banco Hipotecario generaron una nueva expectativa: ser propietario en Mar del Plata. Al compás de esa aspiración tuvo lugar un acelerado proceso de renovación urbana, que hizo familiar la presencia del obrero albañil demoliendo a golpes de piqueta. En un corto lapso el setenta por ciento del casco céntrico quedó convertido en escombros: desaparecieron las mansiones de alrededor de la Plaza y la Avenida Colon y en su lugar se levantaron miles de edificios de departamentos para alojar al vasto universo de clases medias. Con la mudanza de la elite veraneante al Barrio Los Troncos en las cercanías de Playa Grande habría de culminar la secuencia iniciada con los conservadores, el desplazamiento de la 
alta sociedad de sus dominios originales, consolidando el perfil de Mar del Plata como el balneario de todos.A mediados de 1960 ese perfil abarcador e inclusivo irá perdiendo consistencia debido a la deserción de dos grupos importantes, los jóvenes, que preferirán hacer rancho aparte en Villa Gesell, y los sectores más acaudalados, que cruzarán al Uruguay en busca de una playa más selecta en Punta del Este. Mar del Plata ya no habría de ser como antes el balneario de todos pero ciertamente continuaría siendo el balneario de masas del país.

\section{Final}

Una vez que se ha afirmado que la Argentina moderna se desarrolló como país periférico a partir de las riquezas de las vastas llanuras de su litoral, que es una sociedad donde las olas de inmigrantes europeos dejaron una marca indeleble en su población iqué más decir de ella - para completar su perfil- sino que la suya es una sociabilidad formada a partir de la convivencia de un orden jerárquico de prestigio y poder y un empuje igualitario? Así caracterizada, ella no presenta la rigidez de las sociedades señoriales dominantes por mucho tiempo en América Latina, en las que la riqueza y la cuna dan lugar a derechos exclusivos y a la vez permanentes. Precisamente, la función del empuje igualitario ha sido desafiar los privilegios allí adonde se manifestaren, llevando a una mayoría de los argentinos a la creencia en que ninguna persona es por nacimiento inferior a otra y que, por lo tanto, todas están en un pié de igualdad de derechos y aspiraciones sociales. Esta creencia movilizó a lo largo de los años a sucesivas generaciones de argentinos y dio sostén a una experiencia social fuera de lo común en el mundo: un balneario donde los más diversos sectores sociales pudiesen compartir vacaciones de verano en las mismas playas. El veraneo marplatense ocupó, así, un lugar emblemático en nuestra historia: fue la confirmación anual de la promesa de igualdad social que durante muchas décadas vertebró la trayectoria del país. 